\title{
WEIBULL BASED METHODOLOGY FOR CONDITION ASSESSMENT OF CAST IRON WATER MAINS AND ITS APPLICATION
}

\author{
HMS Belmonte $^{\mathrm{a}}$, M Mulheron ${ }^{\mathrm{b}}$, PA Smith ${ }^{\mathrm{b}}$, A Ham ${ }^{\mathrm{c}}$, K Wescombe ${ }^{\mathrm{c}}$ and J Whiter ${ }^{\mathrm{a}}$ \\ a - Thames Water Utilities, Spencer House, Manor Farm Road, Reading, Berks., RG2 0JN, UK \\ b - Faculty of Engineering, University of Surrey, Guildford GU2 7XH, UK. \\ c - Formerly of Thames Water Utilities
}

\begin{abstract}
A qualitative and quantitative understanding of how cast iron water distribution pipes fail in service would facilitate a targeted approach to the management of rehabilitation in the water industry. This paper proposes a technique for assessing the condition of pipes, based on strength characteristics obtained from small samples; this offers an alternative way of estimating the likelihood of failure to current methodologies based on pit-depth measurements.
\end{abstract}

Examination of recovered pipe samples indicates that the strength of the cast iron pipe reduces over time as a result of corrosion, although other time-dependent processes, such as fatigue, may also contribute to this degradation. Taken with previous work, this paper suggests that the variation in strength of small samples removed from cast iron water distribution pipes can be characterised using Weibull methods. It is argued that the Weibull modulus provides a useful indicator of the condition of the pipe. Using scaling arguments, inherent in the Weibull methodology, it is then possible to use data from small samples to predict the likely strength characteristics of water distribution pipes in the ground, which is reasoned to be a good measure of the potential performance of the pipe in service. The Weibull approach is applied to a number of different data sets obtained from testing samples extracted from a range of pipes, which have seen service at various locations in the Thames Water region. One of these data sets was from locations where failure had occurred in service. It is shown that the use of Weibull analysis can identify pipes in the network that have degraded the most significantly. A methodology is suggested whereby this information taken with other performance indicators can be used to identify the local regions where rehabilitation is required most urgently. Alternatively it can be used to identify those regions of the network which are in good condition and unlikely to need repair or replacement work.

\section{Keywords}

Cast iron, Condition Assessment, Pipe, Weibull

\section{Introduction}

The UK water industry operates a supply and distribution network consisting of an ageing, predominantly cast iron, infrastructure. Since Thames Water Utilities took control of its water distribution network in 1989, there have been a significant number of cast iron water distribution pipe bursts associated with the older pipes. A significant proportion of these failures have been in pipes with diameters in the range of 3 - 5 inches; in the majority of the cases these small diameter cast iron pipes failed transversely relative to the pipe length as a result of combinations of flexural and tensile loading. The exact cause of these loads has not been confirmed but ground movement, 
traffic loading, and temperature effects are all possible contributory factors. The bursting of a pipe gives rise to considerable disruption for both the general public and for water utility company, as the pipe needs to be repaired within a short time after the burst event. Most water companies currently operate a managed program to rehabilitate or replace the pipe networks, but even planned rehabilitation and upgrade operations can be disruptive and difficult to arrange. This is particularly true in London, where the majority of Thames Water Utilities network consists of cast iron trunk mains and distribution pipes. As a consequence any rehabilitation strategy needs to be carefully planned. An understanding of how cast iron pipes fail combined with a quantitative understanding of the underlying causal factors likely to induce failure would enhance a targeted approach to rehabilitation planning.

The majority of cast iron distribution pipes currently in service in London were manufactured in various ways some 50 to 150 years ago, so they have a range of compositions, and not surprisingly they exhibit a range of mechanical behaviour. Additionally, the local corrosion environment around each pipe can vary significantly from street to street, or even within a street. This creates a situation where different parts of the network have seen different corrosion rates for different lengths of time, on different types and qualities of cast iron. Hence, in the Thames Water network we can expect there to be a population of pipes with significantly different characteristics, in terms of quality, microstructure, strength, and degree of corrosion. These different pipe characteristics make it very difficult to identify those parts of the network that are in most urgent need of repair and replacement work. Thus condition assessment of cast iron water distribution pipes is an important concern for the water industry.

In order to better understand the in-service failures, it is necessary to have an understanding of the stress to which pipes are subjected and any degradation of mechanical performance with time which might contribute to failure. The stresses are complex and originate from a number of sources, including soil loading, ground surface loading (e.g. due to traffic) and as a result of temperature changes. Regardless of the source of loading, for failure to occur somewhere along a pipe the stress level must exceed the local strength of the pipe, where the local strength may as a result of in-service degradation be significantly less than the initial (as manufactured) strength.

At present the standard water industry method for determining the condition of iron water mains is the "pit depth criterion" (Randall-Smith et al., 1992) ${ }^{2}$. Although the overall approach is consistent across the water industry, the equations used and assumptions applied in determining the condition assessment vary. Generally the method involves taking a half-metre section of pipe, usually removed from service during repair or maintenance work, and shot-blasting it to remove any graphitisation (corrosion product) from both the inner and outer surfaces. The largest pits, on both the interior and exterior of the pipe, are then measured from the corrosion free pipe (i.e. base metal of the pipe), a process which is subject to some uncertainty. A remaining wall thickness can then be calculated by subtracting the largest interior and exterior pits sizes from the average pipe wall thickness (i.e. a worst case scenario). A further step that can be taken is to subtract the minimum wall thickness required to sustain the same pressure for a pipe from its remaining wall thickness. This leads to a parameter termed the "remaining wall thickness to failure". When this parameter has a value of zero, it is argued that there is just sufficient wall thickness remaining in the pipe to allow it to carry the in service pressure. Thus a pass/fail criterion can be made 
from the pit depth data and the remaining wall thickness to failure $(R W T F)$ calculation, pipes that have a $R W T F$ value below zero are considered to have failed the "pit depth criterion".

The pit depth method is attractive in that it is simple to implement, however, there some drawbacks. One issue is that the analysis uses maximum pit depths, in order to represent the worst case condition along a street. The likelihood of finding the maximum pit depth along a street within a short length of pipe is relatively low, so the values used are likely to be an underestimate. Secondly, the pit depth approach is a measure of condition based on the examination of a section of pipe, which does not provide any statistical indication as to the condition of the rest of the pipe. Another potential difficulty, from a fracture mechanics perspective, is that the maximum flaw size may be under-represented by the remaining wall thickness parameter in the pit depth approach. Finally the strengthbased failure criterion that is subsequently applied is simplistic in that it only considers water pressure loading and that is only one element of the stresses on a pipe in service.

In previous papers Atkinson et al. (2002) $)^{3}$ and Belmonte et al. $(2007)^{4}$ it was demonstrated that Weibull analysis could be a useful tool for examining the condition of cast iron water mains. Given that corrosion occurs at apparently random locations on a pipe, associated with material variability and changes in local environmental conditions, any methodology leading to residual life prediction should contain a statistical element. In the next section Weibull methods are examined as a tool for determining condition and performance of cast iron water mains.

\section{The Use of Weibull Methods to Interpret Data from Cast Iron Pipes and Networks}

In a previous paper (Belmonte et al. 20074) Weibull analysis was considered as a method of determining the condition of a cast iron pipe from the strengths of small specimens removed from a section of pipe. It was shown that the Weibull modulus and the characteristic strength are dependent on the microstructure of the cast iron. Weibull modulus and characteristic strength were observed to decrease with increasing graphite flake size, reflecting the larger flake size and flake size distribution inherent in the coarse microstructure.

Using Weibull scaling arguments it is possible (relating both to mode of loading and sample volume) to estimate the strength distribution for the larger pipe from the strength of small samples. In Belmonte et al. (2007) ${ }^{4}$ it was shown that Weibull strength conversions for mode of loading were consistent with experimental results; but there were some problems observed when using the Weibull volume scaling arguments, although these were possibly exacerbated by sampling difficulties.

Weibull analysis is based on measurements of strength; both mean strength and strength variability are influenced by in service degradation (lowering of mean strength and increased strength variability). Hence it is proposed that the Weibull modulus, a measure of the spread of the strength distribution, may be used as a measure of pipe condition and that the strength prediction for a pipe based on Weibull scaling from small sample data may be used as a measure of pipe performance. 
As corrosion is a stochastic process, samples taken from a severely corroded pipe are more likely to have a larger spread of strengths than samples taken from a relatively corrosion-free pipe. Figure 1 shows a schematic plot of cumulative probability against strength for a set of samples taken from "new", "old" or "intermediate" pipes of similar microstructure. The strength distribution for a "new" pipe, one unaffected by corrosion, would be expected to be narrow with a high mean strength, since the strength would be controlled entirely by the inherent flaws (e.g. graphite flakes) present in the cast iron. The strength distribution for an "old" pipe that is severely corroded would be expected to be wide, as the corrosion defects would vary in size across the pipe and the resultant strengths would thus vary depending on the size of the corrosion defects and the mean strength would be lower. An "intermediate" pipe would be one where some parts of the pipe were significantly corroded and failure would occur at a corrosion defect whilst other parts would be relatively corrosion free and fracture would initiate at an inherent defect. This would result in a bimodal strength distribution. Thus a low Weibull modulus can be taken as indicative of a severely degraded pipe with a wide spread of strengths and a high Weibull modulus is indicative of a pipe that is relatively free from degradation.

It is important to note that there is a subtle interplay between the inherent graphite flake size and the degree of corrosion experienced by a cast iron pipe. As it is assumed that the largest sized flaw controls failure, the corrosion defects must therefore be larger than the inherent defects before they dominate failure. In cast iron with a coarse microstructure the corrosion defects need to reach a substantial size (larger than the inherent graphite flake size) before they can dominate failure. However, in a cast iron with a fine microstructure the inherent flaws are smaller and thus a smaller degree of corrosion is necessary before the corrosion defects become large enough to control failure. So a small amount of corrosion on a cast iron with a fine microstructure could have a significant effect on strength, whilst the same degree of corrosion would not have a significant effect on the strength of a cast iron with a coarse microstructure. This means that the significance of the degree of corrosion on a pipe will depend on the microstructure of the cast iron of that pipe.

The degree of deterioration of a pipe is not sufficient measure on which to base an assessment of the likelihood of failure. A degraded pipe (with a low Weibull modulus) will have a higher probability of failure at any given load than a pipe in good condition. Whether or not failure occurs in practice will depend on the in-service loadings acting on that pipe. Indeed a pipe with a low Weibull modulus may have sufficient strength to withstand all the service loadings.

Turning now to a network of pipes, a potentially useful way to characterise its condition is to consider the likely form of the schematic plot of cumulative probability against Weibull modulus, obtained from testing sets of small samples removed from various locations within the network. The form of such a plot is presented in figure 2 for networks consisting of "new" or "old" pipes. It would be expected that a network of new pipes would be characterised by high Weibull moduli with little spread. In comparison, a network of old pipes could be expected to have a larger variation in Weibull modulus, as these pipes would be experiencing a range of conditions. The main driving force for changing the characteristic distribution of a pipe network is time in service and the 
associated degradation due to corrosion. Associated with figure 2, it would also be possible, for a specified probability of failure, to generate a distribution corresponding to the predicted pipe strengths at the various locations in the network where the small sample data have been obtained.

In this paper the bending strengths of small samples taken from pipe sections across the Thames Water distribution network are examined. These bend strength data are examined using Weibull analysis and the Weibull modulus, characteristic strength and predicted full-pipe strengths are then determined for each pipe. The Weibull data and predictions generated are examined and assessed for their usefulness as condition and performance assessment tools.

\section{Experimental}

\subsection{Samples}

\subsubsection{Introduction}

The strength data used in this study was obtained from 266 pipe sections that were exhumed and tested by Thames Water. Data was sampled under three different surveys; a Winter Event program (36 samples), a Random program (113 samples) and a Stratified program (117 samples). Each of the sampling programs is outlined briefly below.

\subsubsection{Winter Event}

During December 2001 and January 2002 there was a significant increase in the number of visible leakage repair work undertaken. This was of interest since a visible leakage increase was observed and associated with a period of cold weather. Although the 2001/2 winter was considered an average winter in terms of weather and pipe burst numbers, the minimum temperature in London dropped to $-1.8^{\circ} \mathrm{C}$.

To ensure that sampling was representative of the failures caused by the winter leakage event, only small diameter ring fractures that had failed during the leakage event period were examined in this study. As the pipes could only be analysed after the event, it was necessary to revisit the appropriate repairs to obtain the pipe samples. From Thames Water records, locations were identified that fit the criteria of the study (i.e. 3-6 inch cast iron mains with a ring fracture that occurred between the $16^{\text {th }}$ of December 2001 and $12^{\text {th }}$ of January 2002). From these it was decided, where possible, to sample ten jobs per water pressure zone. This provided a significant amount of data to describe the leakage event whilst allowing for difficulties in obtaining samples, either physically or due to inaccuracies in the acquired information. It should be noted that all the pipes in the winter event were characterised as pit cast. 
It should be emphasised that the Winter Event sample set is basically a sample set obtained from pipes that fractured (hence failed pipes) during the Winter Leakage Event in 2001/2 across the Thames Water distribution system. The other sample sets are cut-outs from random locations within specified regions.

\subsubsection{Random Program}

The random sampling program was designed to gather information on general asset condition and compare the general condition of water distribution pipes in Metropolitan London (central London), Outer London and Provinces.

Initially for the random sampling program around 40 areas from each performance region (Metropolitan London, Outer London and Provinces) were chosen from areas that had not been previously rehabilitated. From each of these initial 40 areas four streets containing the chosen pipe diameters were selected at random, with a range of street types being selected (from main roads to cul-de-sacs). One sample was obtained for all four streets in each area, giving a total of 120 pipe samples.

The random sampling program was undertaken between August and December 2002, with a total of 125 pipe samples exhumed (although only data from 113 samples were used in this study); 36 were from Metropolitan London ( 7 of which were characterised as spun cast), 43 from Outer London (11 of which were characterised as spun cast) and 34 from Provinces (26 of which were characterised as spun cast). More samples were obtained for the Outer London area due to a change in the performance region's boundaries during the course of the sampling.

\subsubsection{Stratified Program}

The Stratified sampling program was undertaken to sample pipes in areas with good and bad performance histories (in terms of leakage and pipe bursts) in order to further understand the relationship between asset condition and performance.

Each District Metered Area (DMA) within the Metropolitan London performance region were ranked according to their asset repair rate. From this ranking six DMAs were selected, three with a high burst rates and three with a low burst rates. For the high burst rate DMAs, 15 streets with a history of mains repair, and 5 streets with no history of mains repair, were selected. For the low burst rate DMAs, 15 streets with no history of mains repair, and 5 streets with a history of mains repair, were selected.

The stratified sampling programme was undertaken between August and December 2002. A total of 116 pipe samples were exhumed; 43 were from high burst rate streets in high burst rate DMAs (12 of which were characterised as spun cast), 14 were from streets with no significant burst history in high burst rate DMAs ( 2 of which were characterised as spun cast), 46 were from zero burst rate streets in low burst rate DMAs (5 of which were characterised as spun cast) and 13 were from streets with a relatively high burst history in low burst rate 
DMAs in (1 of which was characterised as spun cast). 120 pipe samples had originally been planned for but due to factors such as road closure and traffic management issues resulted in a slight reduction in the number of pipes sampled.

\subsection{Testing}

\subsubsection{Introduction}

A section of pipe was obtained from the location identified by the sampling process by digging down to the pipe and removing a metre section of pipe which was subsequently cut in half. For the Winter Event samples a half metre section was removed from either side of the failure. One of the two half metre sections were then sent for pit depth testing and the other half for four point bend testing. The pit depth testing is an industry standard test (Dempsey and Manook, $1986^{5}$ ).

\subsubsection{Determining the Weibull Parameters}

In the most common application of Weibull statistics, the critical flaw is assumed to lie somewhere within the volume of the sample and the survival probability $\left(P_{s}\right)$ of a volume of material $V$, subjected to a stress, $\sigma$, is given by:

$$
P_{s}(V)=\exp \left[-V\left(\frac{\sigma}{\sigma_{0}}\right)^{m}\right]
$$

The quantity $\sigma_{0}$ is the characteristic strength, which is a material constant (and is defined as the strength when the probability of survival is $\mathrm{e}^{-1}$, or about $37 \%$ ), and $m$, is the Weibull modulus, which is also a material constant and is a measure of the spread of the strength distribution.

The Weibull parameters ( $m$ and $\sigma_{0}$ ) are determined experimentally from tests on a number of samples $N$. The resulting failure strengths are ranked in ascending order and a survival probability is assigned to each sample. The survival probability for the $j^{\text {th }}$ sample in the rank is usually determined, for a small sample set, from:

$$
P_{s}=1-\frac{j-0.375}{N+0.25}
$$

Plotting a graph of $\ln \ln \left(1 / P_{s}\right)$ against the $\ln (\sigma)$ enables the Weibull parameters to be determined. The slope of the linear line of best fit, for the data, is the value of the Weibull modulus while the value of the characteristic strength can be determined from the intercept of the $x$-axis. 
Equations 1 and 2 can be found in most text books (e.g. Davidge ${ }^{6}$ and Watchman ${ }^{7}$ ).

\subsubsection{Flexure Testing}

Small flexure specimens were sectioned from each $0.5 \mathrm{~m}$ length pipe sections that were collected under the different sampling programmes. Where possible ten bend test specimens were taken at various locations around the circumference of the pipe, with the specimens being equally distributed around the four quadrants of the pipe. These specimens were gang milled, with the long length of the specimens parallel to the length of the pipe, to produce parallel-sided bar specimens of material of $10 \mathrm{~mm}$ width and $120 \mathrm{~mm}$ length with depth determined by the pipe wall thickness. The internal and external faces of the specimens were left in the as received condition so as to be representative of the condition of the original pipe. Irregular bearing surfaces were prepared locally to be square to the loading axis by the application of a polyester filler material.

These samples were tested to failure in a four point bending fixture within a $30 \mathrm{kN}$ capacity universal testing machine. The loading rollers were $10 \mathrm{~mm}$ in diameter, with outer rollers spaced $100 \mathrm{~mm}$ apart and inner rollers spaced $50 \mathrm{~mm}$. The samples were oriented such that the face of the bend test specimens associated with the outer surface of the pipe was subject to tension. The specimens were loaded at a constant cross head rate of $0.4 \mathrm{~mm} / \mathrm{min}$. The ultimate load was recorded together with the section width and depth at the fracture face. Fracture strength was calculated using simple bending theory.

The survival probabilities for each specimen of each pipe, in each sample set, were determined according to Equation 2 and the bend strength data obtained were then examined using Weibull analysis and the Weibull modulus and characteristic strength were determined for each pipe. The results for each pipe are presented and discussed in the next section.

\section{Results and Discussion}

The spun and pit cast pipe samples were not separated in this study; however, an examination of the data shows that the Weibull modulus for both the spun and the cast iron exhibit similar ranges (apart from one spun cast iron outlier), with the spun cast iron samples generally exhibiting a higher average strengths.

Table 1. shows the summary of the average strength and Weibull data (Weibull modulus and characteristic strength) results for the winter event sample group. The average strength is the average of the fracture strengths of all the samples tested for that particular pipe. The Weibull modulus is shown, along with the $\mathrm{R}^{2}$ value. It should be noted that each row of data summarises the results of 10 to 15 test samples from a given pipe tested in four point bending. Similar tables showing the results for all the other sample groups can be found in Appendix A.

An analysis of the data suggests that there is no significant difference between the average Weibull modulus for the pit cast and spun cast pipes. This is expected as the Weibull modulus is a measure of the spread of the strength data 
and can be taken to reflect the condition of the pipe. However, both the average and characteristic strengths of the pit cast pipes were generally lower than that of the spun cast pipes. This is thought to reflect the microstructure that results from the different manufacturing processes.

Figure 3 shows a graph of cumulative probability against Weibull modulus for the Random sample set with the Winter Event data also included. It can be seen that the Winter Event data has a much higher percentage of pipes with low Weibull modulus in comparison to the Provinces data. The data from the Metropolitan London and Outer London sets are broadly similar and lie between these two extremes. However below a cumulative probability of 0.5 they can be distinguished with the Metropolitan data having lower Weibull moduli. This makes sense as the Winter Event represents pipes that have failed and thus should be in the "worst" condition. Following this it appears that the Metropolitan London is the next worst region, followed by the Outer London, with the Provinces appearing to be in the best condition.

Figure 4 shows a graph of cumulative probability against Weibull modulus for the Stratified sample set and again the Winter Event data has been included. It can be seen that the Winter Event data has the highest percentage of pipes with low Weibull modulus. It should be noted that the data for the Stratified sample set originates from pipes within Metropolitan London, but do not form part of the Metropolitan London data set presented in this paper. However, all the data sets of the Stratified sample set have similar distributions to that of the Metropolitan London data set. Whilst there is some overlap in the data sets the High Burst Rate DMA, High Burst Rate Streets (High BR, High BR in the key to Figure 4 and 6) region appears to be in worst condition, with the Low Burst Rate DMA, Zero Burst Rate Streets (Low BR, Zero BR in the key to Figure 4 and 6) region appearing to be in the best condition. The number of data points in the data sets for the High Burst Rate DMA, Zero Burst Rate Streets region and Zero Burst Rate DMA, High Burst Rate Streets region are relatively small making it difficult to distinguish them from the other data sets. However, in general the Low Burst Rate DMA performed better than the High Burst Rate DMA.

In section 2 it was mentioned that strength data from a specific geometry under a given mode of loading can be converted by Weibull scaling arguments to an equivalent strength for a given specimen geometry under a different mode of loading. Taking the average failure strengths and the Weibull modulus (obtained by four point bending of small beam samples) for each of the pipes from the different sampling sets, a converted equivalent strength (see Belmonte et al. $(2007)^{4}$ ) was calculated for a typical $3 \mathrm{~m}$ long, $50 \mathrm{~mm}$ radius and $12 \mathrm{~mm}$ thick pipe under threepoint bending.

A three metre long four inch diameter pipe is the standard as manufactured full-length, small diameter, cast iron pipe that was traditionally used as water distribution mains. Three-point bending is used, as it represents the simplest loading condition that a pipe could see in service, assuming the worst case where the pipe was supported at the joints with a surface load being applied along the centre of the pipe. Figures 5 and 6 show graphs of cumulative probability against converted strength using Weibull volume analysis for the Random and Stratified sample sets respectively. 
In figure 5 it can be seen that the Winter Event data has a higher percentage of pipes with lower strength followed by the Metropolitan London data, Outer London data and the Provinces data. The Provinces data has the highest percentage of pipes with high strength. These trends are not unexpected and follow those observed previously in the Weibull modulus data. It also makes intuitive sense that as the Winter Event represents pipes that have failed, and therefore should be in the "worst" condition.

In figure 6 it is again observed that the Winter Event data has a higher percentage of pipes with lower predicted strengths. Again there is some overlap in the data sets, with the High Burst Rate DMA, High Burst Rate Streets region exhibiting the lowest strengths, with the Low Burst Rate DMA, Zero Burst Rate Streets region appearing to be in the best condition, i.e. having the higher strengths. The number of data points in the data sets for the High Burst Rate DMA, Zero Burst Rate Streets region and Zero Burst Rate DMA, High Burst Rate Streets region are relatively small making it difficult to distinguish them from the other data sets. However, in general the Low Burst Rate DMA performed better than the High Burst Rate DMA.

From the foregoing discussion it is suggested that Weibull moduli and predicted pipe strengths extracted from small sample data can provide an indication of the overall condition and likely performance of cast iron water distribution pipes in service. In particular we note that the samples tested from the Winter Event, taken from sites adjacent to where failure has occurred in-service, show the lowest Weibull moduli and the lowest predicted pipe strengths. The data from the Provinces, where comparatively few in service failures occur, show higher Weibull moduli and higher predicted pipe strengths. The Weibull moduli and predicted pipe strength data from the Metropolitan London and Outer London lie between these two extremes, with the data suggesting that the Metropolitan London is in a worse condition. This is consistent with the industry observation that pipes within Metropolitan London region fail more frequently than Outer London.

An ongoing aim of the present work is to develop a pipe condition and performance assessment tool that can be used to assist the industry in identifying those pipes that have degraded the most or alternatively to identify those pipes that are the least degraded. Before considering further how this could be accomplished, it is useful to compare the conclusions that might be drawn from the pipe strength analysis in this paper with the corresponding results from pit depth analysis, which is a current industry standard. From the various locations, the proportion of each data set that failed the industry standard pit depth criterion were $71 \%$ for the Winter Event, $44 \%$ for the Metropolitan London, $24 \%$ for the Outer London and $8 \%$ for the Provinces. In order to determine the proportion of pipes that fail a strength-based criterion, it is necessary to have a value for the in service stress experienced by each pipe. One value put forward by Marshall $(2001)^{8}$ is $90 \mathrm{MPa}$. Although this figure is subject to significant uncertainty, its direct application to the data in figure 5, suggests that $81 \%$ of the Winter Event, $56 \%$ of the Metropolitan London, $42 \%$ of the Outer London and $21 \%$ of the Provinces pipes are at significant risk of failing. We note that there is a similarity in the given percentages of pipes that fail the pit-depth based and strength based criteria, but there is not a one-to-one correlation between the samples that failed the pit depth criterion and those 
that had the lower predicted pipe strengths. For instance, out of the Metropolitan samples, only 10 of the 15 samples that had the lowest strengths also failed the pit depth criterion.

Rather than comparing predicted pipe strength data with a somewhat arbitrary estimate of in-service stress, which will vary for every pipe, an alternative way to use the analyses from the current study would be as two components of a "rules-based" asset assessment model. In such a model, Weibull modulus (a measure of condition) and predicted pipe strength (a measure of potential performance) can both be scored and considered with scores for other parameters indicative of the performance of a particular asset (e.g. asset history, age in service, soil data, utility density, criticality of failure, customer serviceability, seasonal data) in order to give a quantitative measure of the likelihood of failure. This has the potential to provide the industry with a tool to identify those regions within the network that are at the most significant risk of failing and hence in most urgent need of rehabilitation.

\section{Concluding Remarks}

The application of Weibull methods allows the variation in strength of small samples removed from water distribution pipes to be modelled and thereby enable the prediction of pipe strengths using classical scaling arguments. As pipes experience corrosion in service, the mean strength of small samples decreases, due to graphitic corrosion. The Weibull modulus (a measure of spread) decreases as a result of the greater variability of strength associated with corrosion defects. The effect of mode of loading can also be described using Weibull arguments; the strength of a section loaded in tension is predicted to be lower than the strength in bending due to the larger volume of material subjected to tensile stress.

A large number of test data have been analysed, relating to pipe samples from various locations in the Thames Water network. These data show that the pipe samples that failed in the Winter Event have low Weibull moduli and predicted pipe strengths. Further it is shown that the pipe samples for Metropolitan London are of significantly more degraded state than those from Outer London and the Provinces.

Whilst the pit-depth method is experimentally less time consuming the Weibull method models the degradation process more effectively and through the statistical treatment leads to a direct estimate of remaining pipe strength. A direct measure of pipe strength (assessed using total wall thickness, graphitic corrosion and base metal) is perhaps preferable to the indirect method. To formulate a pass/fail design criterion based on the Weibull method requires further work to identify an appropriate value of the calculated pipe strength at which rehabilitation should be carried out. It is suggested that one current estimate of the maximum stress level seen by pipes in service (90 MPa) may be too high, since large portions of the network might be expected to fail under such a high level of stress. Further work is needed to assess the origins and levels of stress in the network more accurately, in particular perhaps those arising from thermal effects.

With regard to targeting for rehabilitation, the Weibull analysis can assist in identifying the regions that are degraded to the greatest extent. These data together with the associated physical description of the underlying 
phenomena can perhaps be used together with historical performance data to identify the local regions where rehabilitation is required most urgently.

\section{Acknowledgements}

The authors would like to acknowledge the contributions to prior work of Ms Natalie Ford and Dr Andrew Prickett at the University of Surrey. We would also like to acknowledge the contribution of Dr Kirsten Atkinson formerly of Thames Water.

The opinions expressed in this paper are those of the authors and not necessarily endorsed by the University of Surrey or Thames Water.

\section{References}

[1] Mulheron, M. and Smith, P.A. (1997) Water Pipe Burst Hypothesis - Recovery and Examination of Repaired 35 in. Pipes. Thames Water Internal Report.

[2] Randall-Smith, M., Russell, A. and Oliphant, R. (1992) Guidance Manual for the Structural Condition Assessment of Trunk Mains. Pipeline Technology, WRc Plc, Swindon.

[3] Atkinson, K., Whiter, J.T., Smith, P.A. and Mulheron, M. (2002) Failure of Small Diameter Cast Iron Pipes. Urban Water, 4(3), pp 263-271.

[4] Belmonte, H.M.S., Mulheron, M. and Smith, P.A. (2007) Weibull Analysis, Extrapolations and Implications for Condition Assessment of Cast Iron Water Mains, Fatigue and Fracture of Engineering Materials and Structures, 30, 964-990.

[5] Dempsey, P. and Manook, B.A. (1986) Assessing the Condition of Cast Iron Pipes, Source Document No.9 for the Water Mains Rehabilitation Manual, WRc Plc, Swindon.

[6] Davidge RW (1979) Mechanical Behaviour of Ceramics, Cambridge University Press, Cambridge, UK.

[7] Wachtman JB (1996) Mechanical Behaviour of Ceramics, John Wiley \& Sons Inc, New York, USA.

[8] Marshall, P. (2001) The Residual Structural Properties of Cast Iron Pipes - Structural and Design Criteria for Linings for Water Mains, Pipeline Innovation, UK Water Industry Research Limited, Report Ref. No. $01 / \mathrm{WM} / 02 / 14$ 
Appendix A - Weibull Modulus Data

In the Tables A1 to A7 the pipe sample reference numbers that have an asterisk are pipes that have been identified as being spun cast, whilst those being pit cast. 


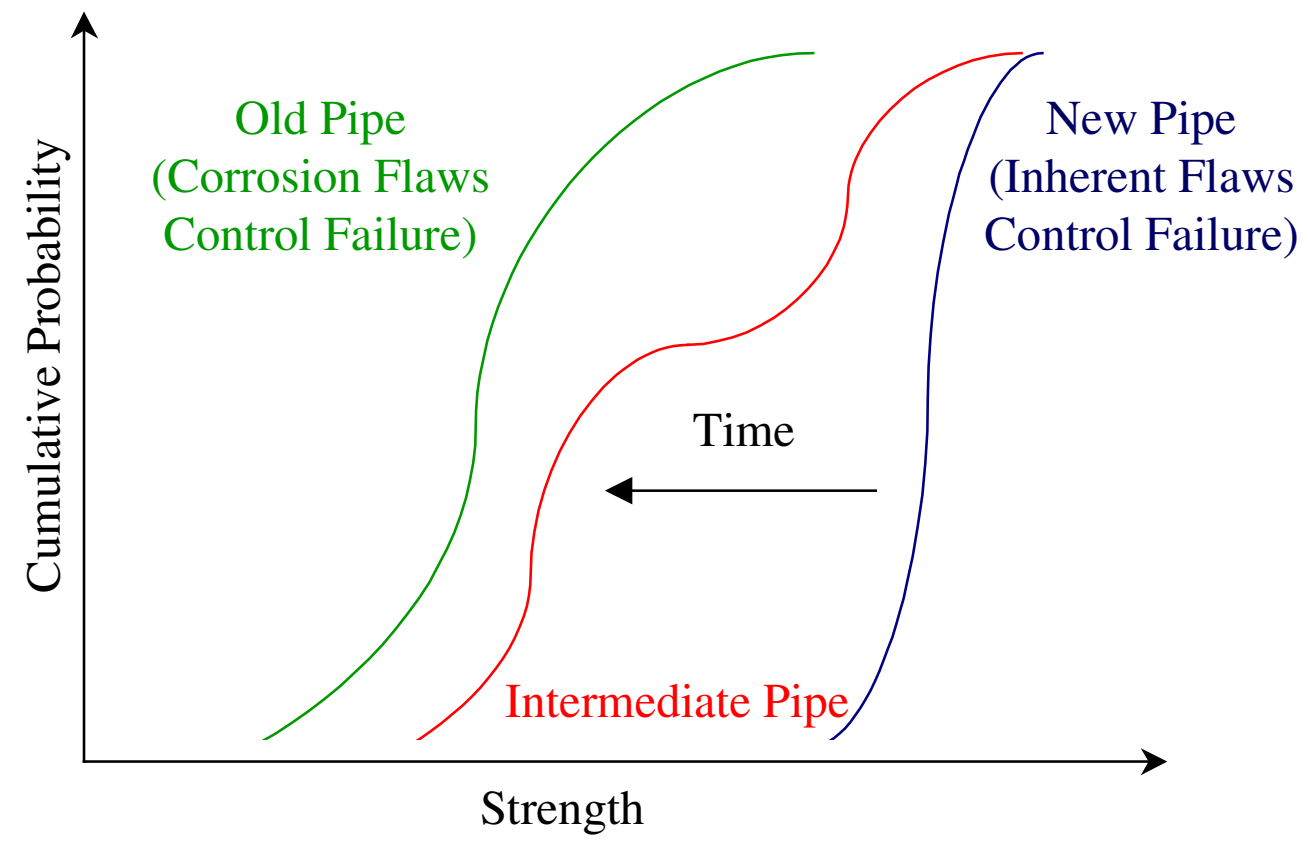

Fig. 1 Schematic Cumulative Probability Distributions of Strength for New, Intermediate and Old Pipe. 


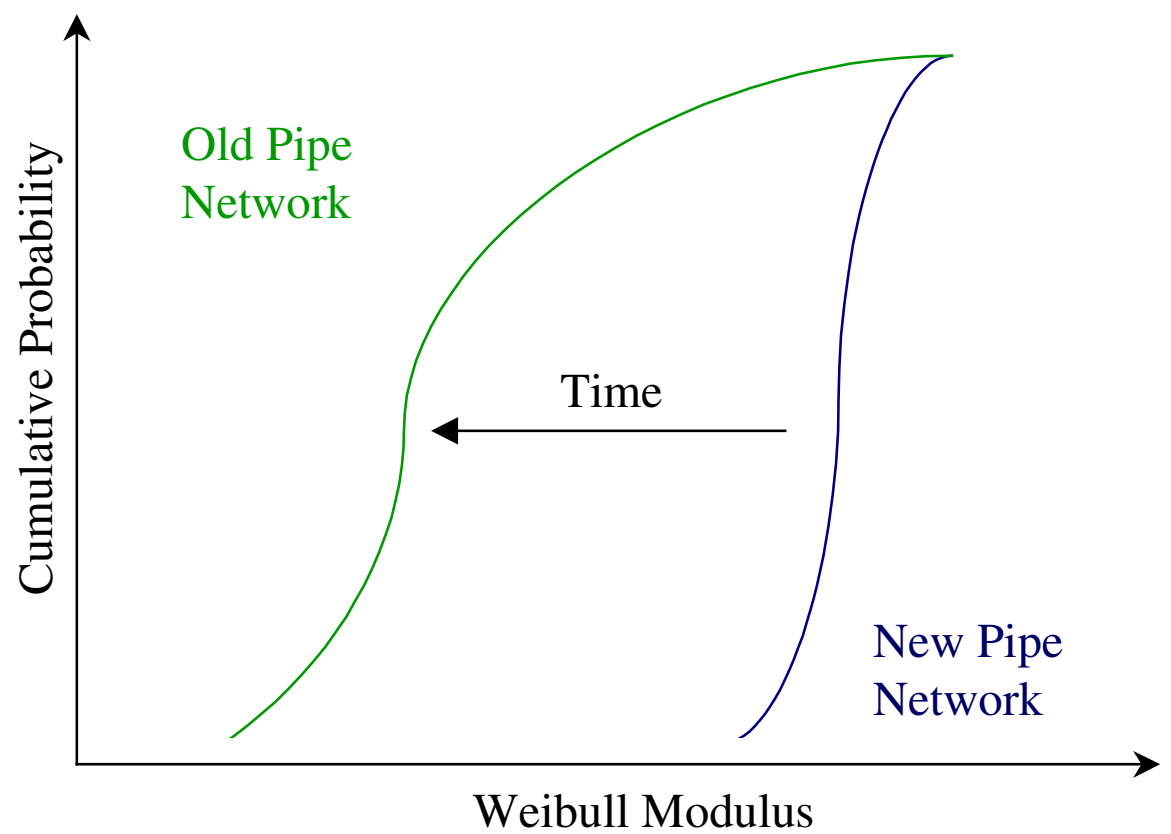

Fig. 2 Schematic Cumulative Probability Distributions of Weibull Modulus for Pipes from New and Old Networks. 


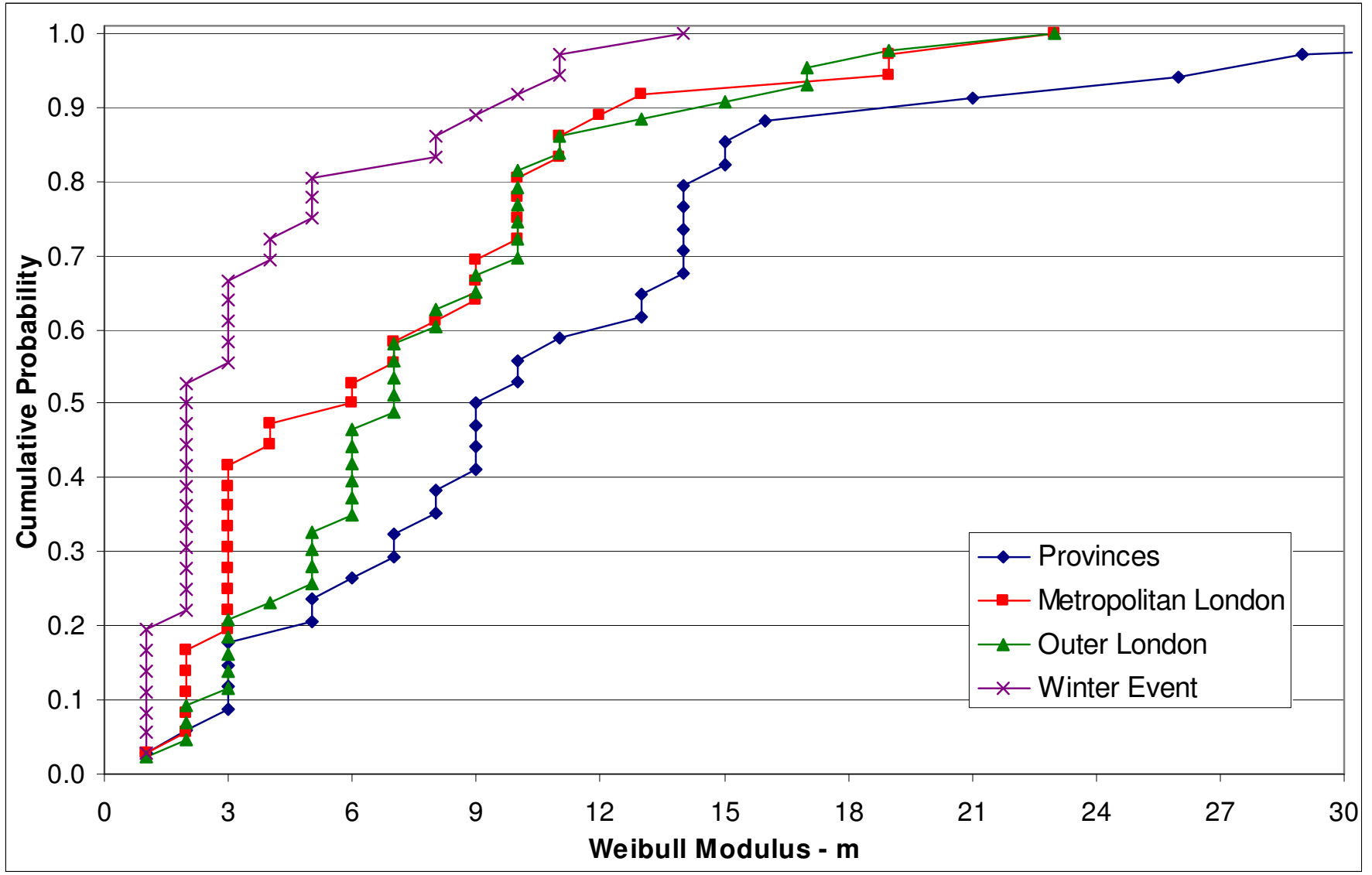

Fig. 3 Cumulative Probability Distribution of the Weibull Modulus for the Random Sample Set. 


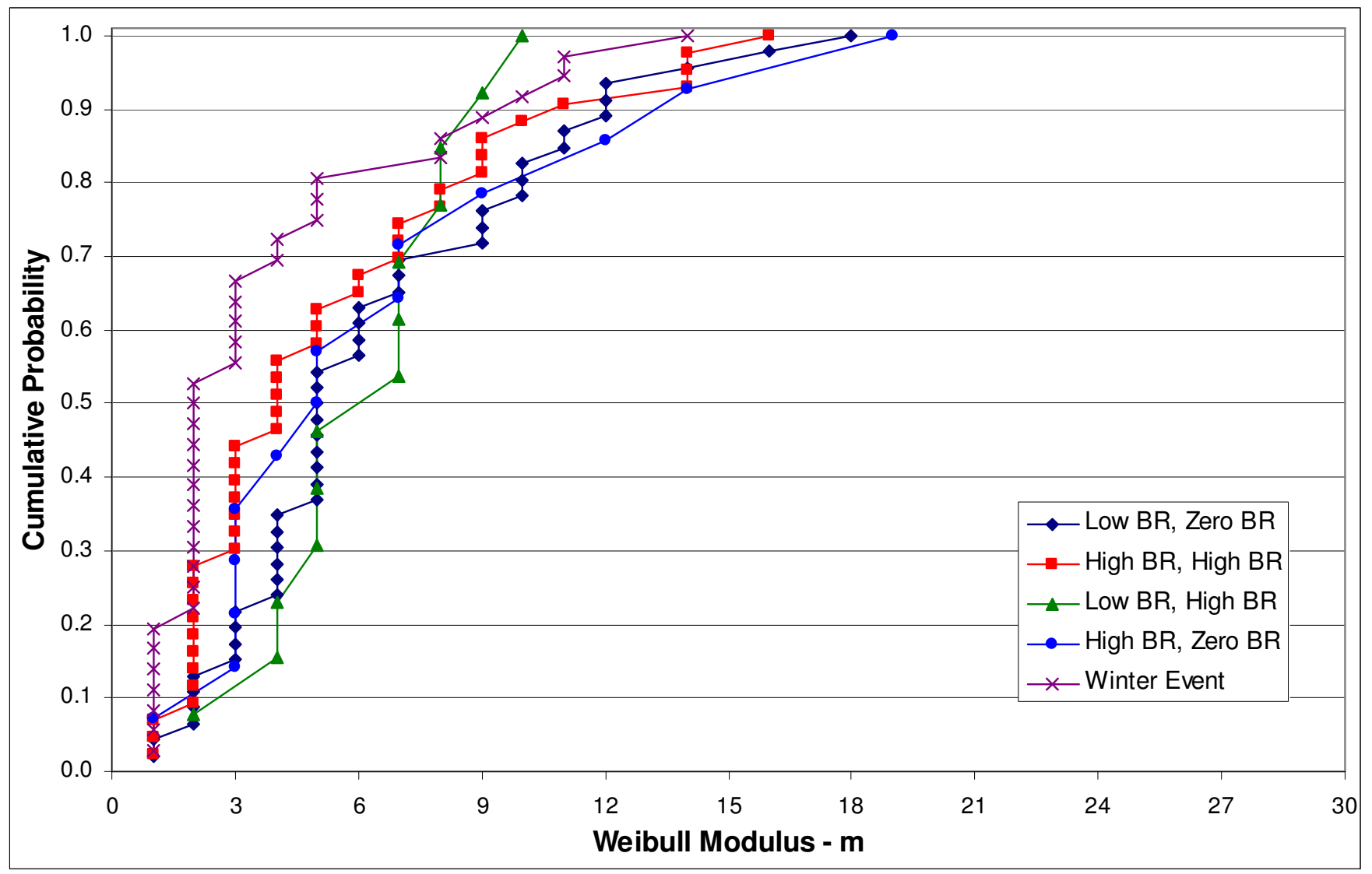

Fig. 4 Cumulative Probability Distribution of the Weibull Modulus for the Stratified Sample Set. 


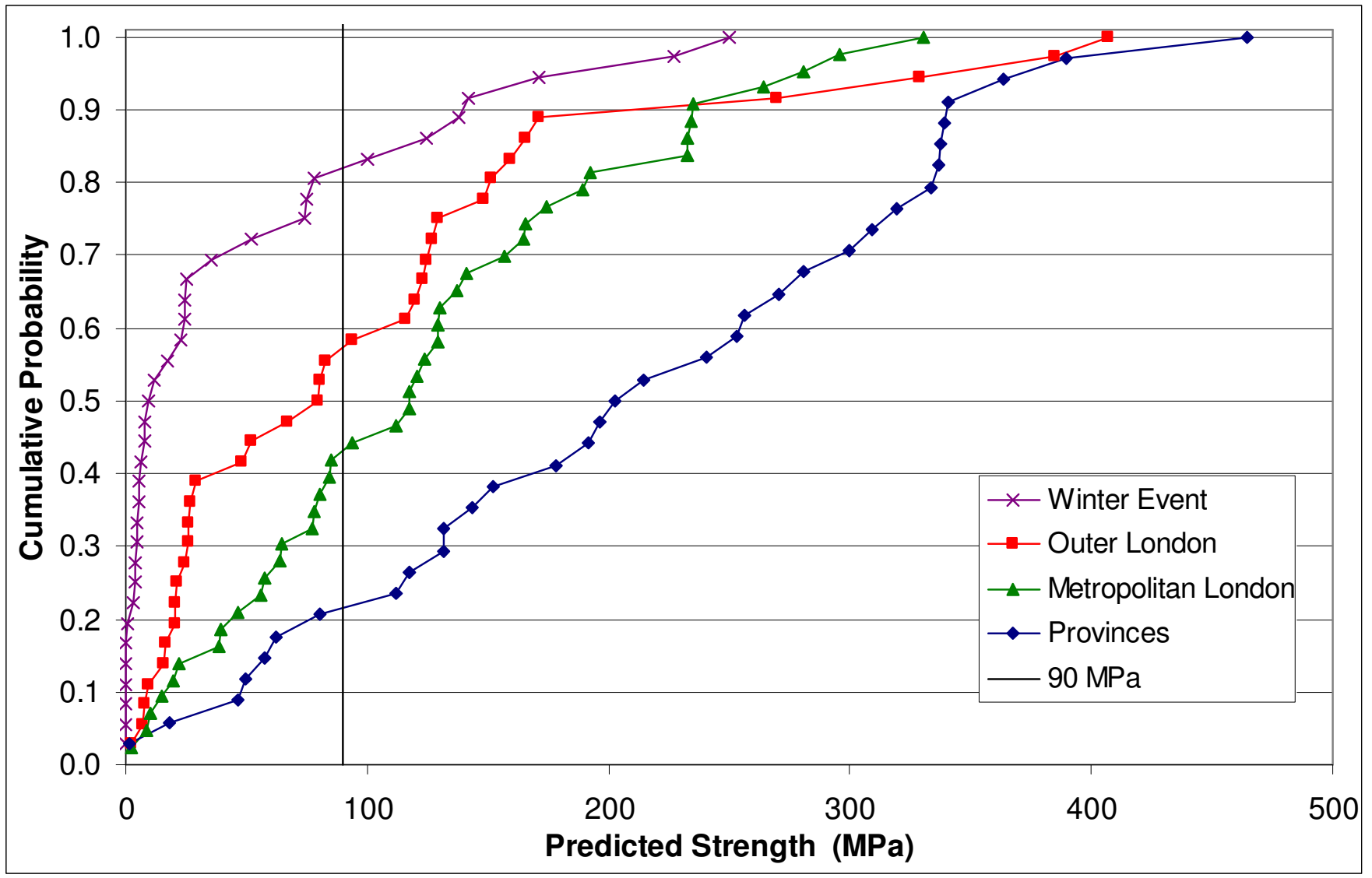

Fig. 5 Cumulative Probability Distribution of Predicted Pipe Strength Determined from Weibull Volume Analysis for the Random Sample Set. 


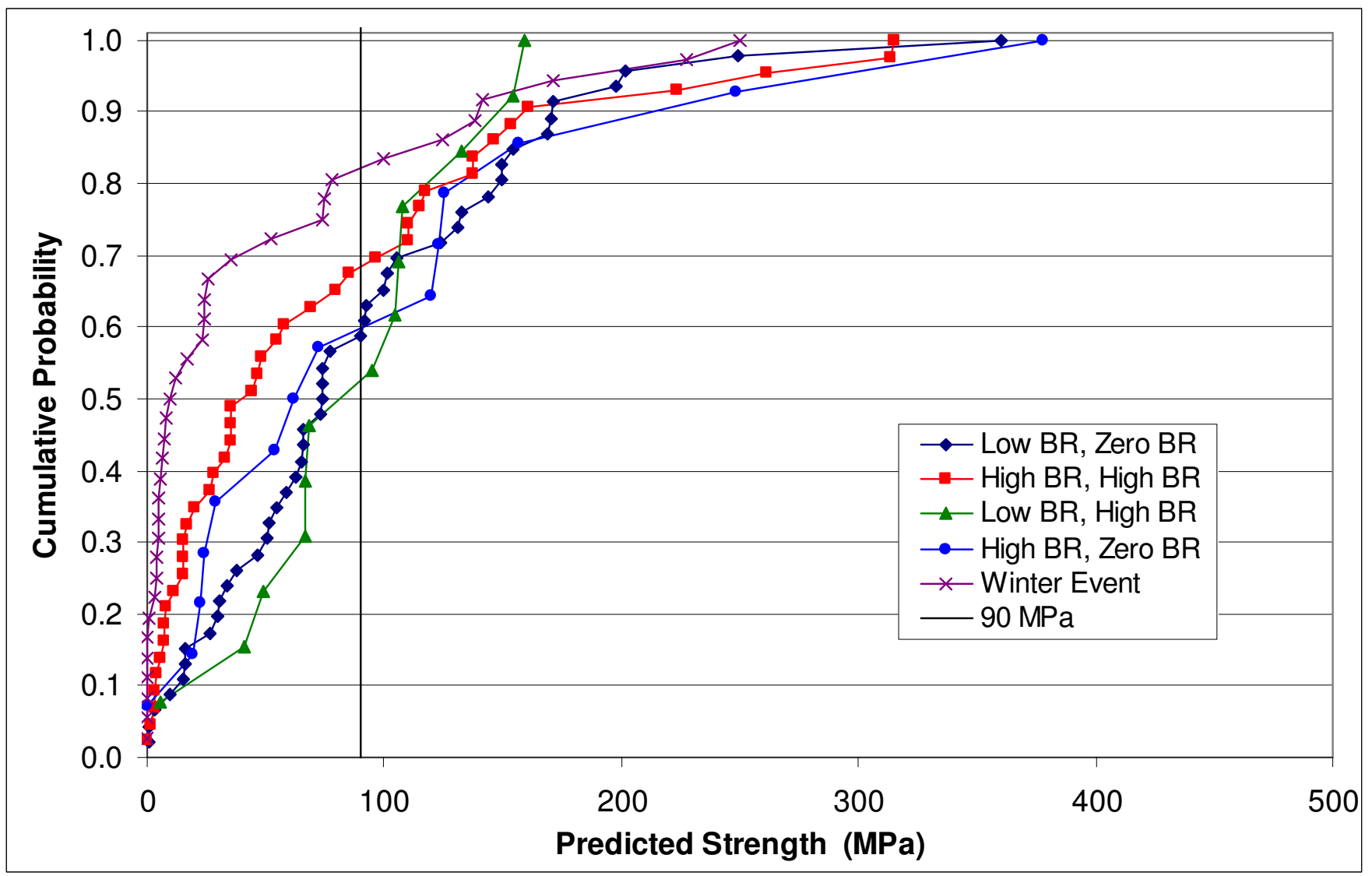

Fig. 6 Cumulative Probability Distribution of Predicted Pipe Strength Determined from Weibull Volume Analysis for the Stratified Sample Set. 
Table 1. Summary of Weibull Data for the36 Pipe Samples Collected Under The Winter Event Programme.

\begin{tabular}{|c|c|c|c|c|}
\hline SMS Sample Ref. & Average Strength & Weibull Modulus & Char. Strength & 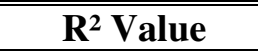 \\
\hline & $\sigma$ & $m$ & $\sigma_{0}$ & $\mathrm{R}^{2}$ \\
\hline & (MPa) & (Dimensionless) & (MPa) & (Dimensionless) \\
\hline EE5384 & 129.4 & 2 & 148.2 & 0.89 \\
\hline EE5385 & 191.6 & 2 & 219.3 & 0.93 \\
\hline EE5400 & 282.1 & 2 & 323.8 & 0.99 \\
\hline EE5401 & 206.2 & 11 & 215.2 & 0.92 \\
\hline EE5409 & 251.6 & 14 & 260.7 & 0.95 \\
\hline EE5386 & 157.1 & 4 & 173.0 & 0.93 \\
\hline EE5390 & 461.5 & 8 & 489.2 & 0.92 \\
\hline EE5414 & 68.3 & 2 & 77.7 & 0.86 \\
\hline EE5415 & 190.4 & 3 & 219.1 & 0.81 \\
\hline EE5382 & 186.7 & 3 & 209.5 & 0.92 \\
\hline EE5403 & 177.0 & 2 & 201.0 & 0.97 \\
\hline EE5383 & 281.5 & 8 & 297.6 & 0.98 \\
\hline EE5444 & 186.4 & 9 & 196.7 & 0.92 \\
\hline EE5445 & 92.4 & 1 & 111.0 & 0.89 \\
\hline EE5437 & 218.6 & 2 & 261.2 & 0.86 \\
\hline EE5387 & 132.3 & 3 & 148.5 & 0.95 \\
\hline EE5388 & 413.4 & 11 & 432.2 & 0.92 \\
\hline EE5404 & 168.9 & 5 & 183.8 & 0.96 \\
\hline EE5406 & 119.9 & 2 & 135.7 & 0.98 \\
\hline EE5408 & 143.1 & 2 & 163.8 & 0.96 \\
\hline EE5411 & 99.5 & 2 & 119.0 & 0.90 \\
\hline EE5428 & 114.6 & 1 & 104.5 & 0.98 \\
\hline EE5436 & 118.6 & 2 & 137.9 & 0.94 \\
\hline EE5405 & 200.0 & 1 & 238.6 & 0.92 \\
\hline EE5407 & 387.7 & 1 & 493.4 & 0.81 \\
\hline EE5412 & 240.2 & 5 & 262.0 & 0.85 \\
\hline EE5417 & 177.5 & 3 & 198.6 & 0.98 \\
\hline EE5389 & 109.3 & 1 & 123.9 & 0.93 \\
\hline EE5410 & 187.2 & 1 & 220.7 & 0.92 \\
\hline EE5402 & 83.7 & 2 & 97.3 & 0.91 \\
\hline EE5413 & 242.4 & 5 & 264.2 & 0.92 \\
\hline EE5435 & 347.4 & 4 & 383.3 & 0.98 \\
\hline EE5391 & 185.0 & 3 & 209.4 & 0.96 \\
\hline EE5434 & 98.3 & 1 & 110.5 & 0.94 \\
\hline EE5347 & 91.6 & 2 & 109.1 & 0.42 \\
\hline EE5347 & 247.3 & 10 & 259.5 & 0.98 \\
\hline
\end{tabular}


Table A1. Summary of Weibull Data for the 34 Pipe Samples Collected Under The Random Sampling Programme for the Provinces.

\begin{tabular}{|c|c|c|c|c|}
\hline TWA Sample Ref. & Average Strength & "Weibull Modulus & Char. Strength & $\mathbf{R}^{2}$ Value \\
\hline & $\sigma$ & $m$ & $\sigma_{0}$ & $\mathrm{R}^{2}$ \\
\hline & (MPa) & (Dimensionless) & (MPa) & (Dimensionless) \\
\hline 9-002* & 396.7 & 8 & 420.7 & 0.97 \\
\hline 9-003* & 434.7 & 21 & 445.4 & 0.81 \\
\hline 9-004* & 458.7 & 15 & 474.3 & 0.89 \\
\hline 9-008 & 357.4 & 3 & 401.7 & 0.87 \\
\hline 9-009* & 267.7 & 5 & 291.7 & 0.96 \\
\hline 9-010* & 400.7 & 14 & 415.3 & 0.87 \\
\hline 9-011* & 448.9 & 6 & 482.2 & 0.92 \\
\hline $9-012 *$ & 472.0 & 7 & 519.9 & 0.96 \\
\hline 9-013* & 354.7 & 14 & 367.4 & 0.98 \\
\hline 9-036* & 401.5 & 29 & 408.7 & 0.92 \\
\hline $9-055^{*}$ & 422.5 & 16 & 436.1 & 0.94 \\
\hline $9-056$ & 242.7 & 9 & 256.2 & 0.91 \\
\hline 9-057* & 416.1 & 42 & 421.3 & 0.97 \\
\hline $9-058$ & 218.6 & 8 & 231.9 & 0.92 \\
\hline $9-092$ & 350.9 & 3 & 392.8 & 0.98 \\
\hline $9-094 *$ & 487.3 & 11 & 508.7 & 0.94 \\
\hline $9-095^{*}$ & 474.6 & 10 & 498.2 & 0.87 \\
\hline $9-096$ & 348.3 & 1 & 447.2 & 0.77 \\
\hline 9-113* & 510.4 & 14 & 529.3 & 0.95 \\
\hline 9-114* & 424.1 & 13 & 440.9 & 0.90 \\
\hline 9-124* & 421.5 & 13 & 437.3 & 0.96 \\
\hline $9-125^{*}$ & 575.5 & 26 & 587.1 & 0.97 \\
\hline $9-126^{*}$ & 580.5 & 14 & 601.8 & 0.94 \\
\hline $9-156^{*}$ & 416.4 & 2 & 494.3 & 0.86 \\
\hline $9-157 *$ & 429.0 & 7 & 456.9 & 0.91 \\
\hline 9-196* & 435.1 & 3 & 499.4 & 0.84 \\
\hline 9-207 & 216.2 & 9 & 228.4 & 0.90 \\
\hline $9-208$ & 273.8 & 10 & 287.8 & 0.94 \\
\hline $9-233 *$ & 414.6 & 5 & 450.5 & 0.96 \\
\hline $9-234 *$ & 383.6 & 3 & 433.5 & 0.97 \\
\hline $9-235^{*}$ & 387.8 & 9 & 407.6 & 0.96 \\
\hline 9-243* & 481.7 & 15 & 498.3 & 0.94 \\
\hline 9-245* & 502.8 & 14 & 520.8 & 0.94 \\
\hline $9-252$ & 260.6 & 9 & 274.6 & 0.73 \\
\hline
\end{tabular}


Table A2. Summary of Weibull Data for the 36 Pipe Samples Collected Under The Random Sampling Programme for Metropolitan London.

\begin{tabular}{|c|c|c|c|c|}
\hline TWA Sample Ref. & Average Strength & Weibull Modulus & Char. Strength & $\mathbf{R}^{2}$ Value \\
\hline & $\sigma$ & $m$ & $\sigma_{0}$ & $\mathrm{R}^{2}$ \\
\hline & $(\mathrm{MPa})$ & (Dimensionless) & $(\mathrm{MPa})$ & (Dimensionless) \\
\hline $9-021$ & 289.9 & 10 & 304.2 & 0.91 \\
\hline $9-022$ & 187.5 & 11 & 195.6 & 0.98 \\
\hline $9-023$ & 348.4 & 4 & 386.5 & 0.87 \\
\hline 9-024 & 124.5 & 3 & 140.6 & 0.91 \\
\hline 9-031 & 228.9 & 9 & 241.2 & 0.94 \\
\hline $9-032^{*}$ & 223.2 & 10 & 234.3 & 0.86 \\
\hline $9-033$ & 234.5 & 8 & 247.9 & 0.91 \\
\hline $9-034$ & 141.2 & 3 & 159.1 & 0.96 \\
\hline $9-035$ & 268.6 & 12 & 279.8 & 0.68 \\
\hline $9-037$ & 209.1 & 3 & 237.9 & 0.89 \\
\hline $9-040$ & 129.7 & 1 & 147.7 & 0.99 \\
\hline 9-101* & 452.1 & 13 & 469.9 & 0.91 \\
\hline 9-118 & 219.5 & 4 & 242.1 & 0.95 \\
\hline $9-134$ & 438.1 & 19 & 450.0 & 0.70 \\
\hline $9-145$ & 187.3 & 3 & 212.3 & 0.88 \\
\hline $9-146$ & 246.6 & 10 & 258.3 & 0.91 \\
\hline $9-148^{*}$ & 238.3 & 9 & 250.4 & 0.94 \\
\hline $9-164$ & 146.9 & 2 & 167.1 & 0.96 \\
\hline $9-165$ & 229.2 & 3 & 257.4 & 0.88 \\
\hline $9-168^{*}$ & 521.8 & 23 & 533.7 & 0.87 \\
\hline $9-173$ & 138.1 & 3 & 155.1 & 0.90 \\
\hline 9-186 & 270.6 & 2 & 320.8 & 0.69 \\
\hline $9-200$ & 204.1 & 7 & 217.5 & 0.88 \\
\hline $9-202$ & 215.1 & 6 & 231.4 & 0.92 \\
\hline $9-215$ & 268.4 & 10 & 281.9 & 0.93 \\
\hline $9-219^{*}$ & 183.2 & 6 & 198.1 & 0.74 \\
\hline $9-227$ & 532.5 & 19 & 546.5 & 0.94 \\
\hline $9-228$ & 351.6 & 2 & 418.4 & 0.84 \\
\hline $9-230^{*}$ & 147.9 & 2 & 172.8 & 0.92 \\
\hline $9-237$ & 185.7 & 3 & 207.6 & 0.97 \\
\hline 9-239* & 122.5 & 2 & 139.2 & 0.98 \\
\hline $9-246$ & 266.1 & 11 & 278.2 & 0.90 \\
\hline $9-247$ & 191.4 & 3 & 219.4 & 0.89 \\
\hline $9-248$ & 243.0 & 9 & 256.4 & 0.85 \\
\hline $9-251$ & 200.1 & 3 & 226.5 & 0.93 \\
\hline $9-253$ & 195.0 & 7 & 209.0 & 0.90 \\
\hline
\end{tabular}


Table A3. Summary of Weibull Data for the 43 Pipe Samples Collected Under The Random Sampling Programme for Outer London.

\begin{tabular}{|c|c|c|c|c|}
\hline TWA Sample Ref. & Average Strength & Weibull Modulus & Char. Strength & $\overline{\mathbf{R}^{2} \text { Value }}$ \\
\hline & $\sigma$ & $m$ & $\sigma_{0}$ & $\mathrm{R}^{2}$ \\
\hline & $(\mathrm{MPa})$ & (Dimensionless) & $(\mathrm{MPa})$ & (Dimensionless) \\
\hline 9-001 & 211.1 & 10 & 221.8 & $\begin{array}{c}0.92 \\
\end{array}$ \\
\hline $9-015$ & 151.5 & 3 & 171.2 & 0.88 \\
\hline $9-016$ & 242.1 & 6 & 260.3 & 0.93 \\
\hline 9-017 & 230.0 & 10 & 241.4 & 0.97 \\
\hline 9-018 & 279.3 & 7 & 298.2 & 0.97 \\
\hline 9-019* & 436.0 & 5 & 477.3 & 0.73 \\
\hline $9-020$ & 211.7 & 1 & 271.7 & 0.70 \\
\hline $9-025$ & 227.8 & 2 & 271.6 & 0.81 \\
\hline $9-026$ & 197.4 & 2 & 236.3 & 0.83 \\
\hline 9-027* & 446.4 & 10 & 468.7 & 0.94 \\
\hline $9-028^{*}$ & 469.5 & 11 & 490.7 & 0.97 \\
\hline $9-029$ & 182.2 & 2 & 207.9 & 0.88 \\
\hline 9-030 & 237.2 & 17 & 244.0 & 0.98 \\
\hline $9-038$ & 121.1 & 3 & 136.7 & 0.95 \\
\hline $9-041$ & 223.0 & 6 & 241.2 & 0.96 \\
\hline $9-048$ & 254.6 & 9 & 268.2 & 0.96 \\
\hline $9-050$ & 184.6 & 6 & 199.8 & 0.90 \\
\hline $9-051$ & 207.6 & 3 & 231.5 & 0.95 \\
\hline $9-052^{*}$ & 194.3 & 6 & 209.3 & 0.87 \\
\hline $9-053$ & 259.8 & 19 & 266.7 & 0.85 \\
\hline 9-054* & 471.1 & 17 & 485.0 & 0.93 \\
\hline 9-097 & 283.6 & 7 & 301.7 & 0.90 \\
\hline 9-098* & 441.2 & 8 & 466.8 & 0.99 \\
\hline 9-099* & 509.4 & 5 & 552.9 & 0.87 \\
\hline $9-100$ & 170.0 & 5 & 184.1 & 0.95 \\
\hline $9-103$ & 184.5 & 7 & 197.1 & 0.94 \\
\hline 9-104* & 262.2 & 4 & 292.1 & 0.94 \\
\hline $9-128^{*}$ & 456.7 & 9 & 482.0 & 0.83 \\
\hline $9-129$ & 235.4 & 15 & 243.5 & 0.95 \\
\hline $9-130$ & 214.3 & 10 & 225.1 & 0.96 \\
\hline 9-135 & 265.8 & 3 & 301.8 & 0.86 \\
\hline $9-143$ & 208.5 & 6 & 224.2 & 0.96 \\
\hline 9-147* & 511.0 & 10 & 535.6 & 0.88 \\
\hline 9-158 & 238.7 & 6 & 259.0 & 0.86 \\
\hline $9-159$ & 292.8 & 23 & 299.2 & 0.97 \\
\hline $9-160$ & 268.3 & 13 & 278.7 & 0.88 \\
\hline $9-161$ & 365.5 & 7 & 389.9 & 0.82 \\
\hline $9-162$ & 265.4 & 8 & 281.2 & 0.92 \\
\hline 9-171* & 479.7 & 10 & 504.0 & 0.88 \\
\hline $9-174$ & 235.3 & 11 & 245.9 & 0.98 \\
\hline $9-201$ & 253.4 & 7 & 271.0 & 0.89 \\
\hline $9-216$ & 224.2 & 3 & 251.9 & 0.79 \\
\hline $9-218$ & 206.5 & 5 & 225.9 & 0.87 \\
\hline
\end{tabular}


Table A4. Summary of Weibull Data for the 46 Pipe Samples Collected Under The Stratified Sampling Programme for Low Burst Rate DMA, Zero Bust Rate Streets.

\begin{tabular}{|c|c|c|c|c|}
\hline TWA Sample Ref. & Average Strength & Weibull Modulus & Char. Strength & $\overline{\mathbf{R}^{2} \text { Value }}$ \\
\hline & $\sigma$ & $m$ & $\sigma_{0}$ & $\mathrm{R}^{2}$ \\
\hline & $(\mathrm{MPa})$ & (Dimensionless) & $(\mathrm{MPa})$ & (Dimensionless) \\
\hline 9-042 & 195.7 & 2 & 225.7 & $\begin{array}{c}0.97 \\
\end{array}$ \\
\hline $9-043$ & 200.8 & 5 & 218.1 & 0.98 \\
\hline $9-049$ & 189.3 & 3 & 212.8 & 0.93 \\
\hline 9-064 & 202.7 & 4 & 222.6 & 0.95 \\
\hline $9-065$ & 280.1 & 16 & 289.2 & 0.86 \\
\hline $9-066$ & 213.7 & 4 & 236.1 & 0.92 \\
\hline $9-067$ & 254.2 & 14 & 263.7 & 0.77 \\
\hline $9-068$ & 136.0 & 4 & 149.9 & 0.94 \\
\hline $9-069$ & 178.7 & 4 & 198.1 & 0.96 \\
\hline $9-070$ & 88.2 & 1 & 99.5 & 0.97 \\
\hline $9-071$ & 297.3 & 12 & 310.0 & 0.94 \\
\hline $9-072$ & 218.8 & 5 & 238.0 & 0.93 \\
\hline $9-073$ & 182.4 & 9 & 192.7 & 0.82 \\
\hline $9-076$ & 227.0 & 10 & 237.5 & 0.97 \\
\hline 9-079 & 176.9 & 2 & 203.2 & 0.90 \\
\hline 9-082 & 216.5 & 5 & 234.5 & 0.97 \\
\hline $9-084$ & 193.3 & 4 & 216.3 & 0.90 \\
\hline $9-085$ & 232.8 & 2 & 268.3 & 0.94 \\
\hline $9-086$ & 165.0 & 1 & 238.8 & 0.55 \\
\hline 9-087* & 498.8 & 18 & 512.6 & 0.90 \\
\hline 9-106 & 223.1 & 4 & 247.4 & 0.90 \\
\hline $9-107$ & 183.9 & 3 & 211.4 & 0.86 \\
\hline 9-108 & 217.9 & 7 & 232.7 & 0.85 \\
\hline $9-110$ & 235.9 & 12 & 246.0 & 0.86 \\
\hline 9-111* & 420.0 & 3 & 470.6 & 0.95 \\
\hline $9-112$ & 215.6 & 6 & 232.6 & 0.95 \\
\hline $9-131$ & 224.5 & 5 & 244.2 & 0.89 \\
\hline $9-138$ & 266.0 & 12 & 276.3 & 0.97 \\
\hline $9-151$ & 187.8 & 6 & 201.6 & 0.94 \\
\hline 9-167 & 252.7 & 11 & 263.8 & 0.93 \\
\hline 9-176 & 228.2 & 9 & 240.4 & 0.86 \\
\hline 9-177 & 441.4 & 7 & 470.0 & 0.94 \\
\hline 9-182 & 200.0 & 5 & 217.0 & 0.91 \\
\hline 9-187 & 246.2 & 11 & 257.1 & 0.92 \\
\hline 9-189 & 247.3 & 10 & 259.0 & 0.97 \\
\hline 9-191* & 109.0 & 2 & 130.5 & 0.87 \\
\hline $9-205$ & 174.7 & 5 & 189.2 & 0.96 \\
\hline $9-209$ & 198.1 & 5 & 214.7 & 0.85 \\
\hline 9-217* & 231.7 & 10 & 243.2 & 0.98 \\
\hline $9-220^{*}$ & 477.8 & 9 & 504.4 & 0.93 \\
\hline $9-222$ & 275.2 & 5 & 299.7 & 0.82 \\
\hline $9-223$ & 237.2 & 6 & 255.1 & 0.94 \\
\hline $9-225$ & 202.3 & 3 & 227.5 & 0.94 \\
\hline $9-231$ & 227.0 & 7 & 241.8 & 0.99 \\
\hline $9-232$ & 198.5 & 5 & 217.4 & 0.97 \\
\hline $9-250$ & 258.5 & 6 & 278.4 & 0.71 \\
\hline
\end{tabular}


Table A5. Summary of Weibull Data for the 43 Pipe Samples Collected Under The Stratified Sampling Programme for High Burst Rate DMA, High Burst Rate Streets.

\begin{tabular}{|c|c|c|c|c|}
\hline TWA Sample Ref. & Average Strength & Weibull Modulus & Char. Strength & $\overline{\mathbf{R}^{2} \text { Value }}$ \\
\hline & $\sigma$ & $m$ & $\sigma_{0}$ & $\mathrm{R}^{2}$ \\
\hline & $(\mathrm{MPa})$ & (Dimensionless) & $(\mathrm{MPa})$ & (Dimensionless) \\
\hline $9-045$ & 245.2 & 111 & 256.4 & 0.96 \\
\hline 9-074* & 353.1 & 2 & 419.3 & 0.91 \\
\hline $9-075^{*}$ & 81.5 & 2 & 94.4 & 0.93 \\
\hline 9-077 & 93.2 & 4 & 103.1 & 0.95 \\
\hline 9-078* & 164.4 & 3 & 183.7 & 0.97 \\
\hline 9-088 & 184.7 & 5 & 202.4 & 0.94 \\
\hline 9-089 & 215.5 & 5 & 235.9 & 0.96 \\
\hline 9-091* & 406.9 & 1 & 524.8 & 0.73 \\
\hline 9-117* & 182.4 & 4 & 203.4 & 0.90 \\
\hline $9-119$ & 292.0 & 9 & 308.0 & 0.92 \\
\hline $9-120^{*}$ & 504.2 & 7 & 537.2 & 0.95 \\
\hline $9-123$ & 120.4 & 2 & 143.5 & 0.92 \\
\hline $9-132$ & 162.2 & 2 & 188.5 & 0.86 \\
\hline $9-133$ & 219.6 & 6 & 236.5 & 0.94 \\
\hline $9-136$ & 245.7 & 10 & 258.1 & 0.94 \\
\hline $9-137$ & 229.0 & 8 & 242.0 & 0.97 \\
\hline 9-139* & 169.5 & 3 & 192.7 & 0.90 \\
\hline $9-141$ & 182.7 & 2 & 209.2 & 0.90 \\
\hline $9-142$ & 103.7 & 2 & 121.0 & 0.81 \\
\hline $9-150^{*}$ & 207.1 & 2 & 239.2 & 0.91 \\
\hline $9-153$ & 137.4 & 1 & 158.2 & 0.96 \\
\hline 9-154 & 229.6 & 6 & 246.5 & 0.92 \\
\hline $9-166$ & 197.3 & 16 & 203.6 & 0.92 \\
\hline $9-172$ & 245.3 & 8 & 260.1 & 0.96 \\
\hline $9-175$ & 198.8 & 3 & 223.2 & 0.98 \\
\hline $9-179$ & 142.9 & 1 & 184.7 & 0.80 \\
\hline $9-180$ & 230.8 & 4 & 258.5 & 0.88 \\
\hline $9-181$ & 218.0 & 5 & 237.0 & 0.93 \\
\hline $9-183$ & 261.6 & 3 & 300.6 & 0.71 \\
\hline $9-185$ & 136.8 & 3 & 153.6 & 0.95 \\
\hline $9-190$ & 185.5 & 2 & 213.5 & 0.93 \\
\hline 9-192 & 243.0 & 9 & 256.6 & 0.90 \\
\hline $9-194$ & 184.1 & 4 & 203.0 & 0.97 \\
\hline $9-195$ & 242.9 & 7 & 260.1 & 0.94 \\
\hline $9-204$ & 461.8 & 14 & 478.3 & 0.94 \\
\hline 9-206* & 386.4 & 14 & 400.4 & 0.89 \\
\hline $9-210$ & 260.6 & 7 & 278.9 & 0.90 \\
\hline $9-211$ & 230.1 & 3 & 259.6 & 0.91 \\
\hline $9-212^{*}$ & 166.4 & 4 & 186.4 & 0.82 \\
\hline $9-213$ & 169.5 & 2 & 194.4 & 0.95 \\
\hline $9-214$ & 292.4 & 9 & 307.5 & 0.95 \\
\hline $9-240^{*}$ & 323.5 & 3 & 364.0 & 0.84 \\
\hline $9-241^{*}$ & 465.5 & 14 & 482.3 & 0.95 \\
\hline
\end{tabular}


Table A6. Summary of Weibull Data for the 13 Pipe Samples Collected Under The Stratified Sampling Programme for Low Burst Rate DMA, High Burst Rate Streets.

\begin{tabular}{|c|c|c|c|c|}
\hline TWA Sample Ref. & Average Strength & Weibull Modulus & Char. Strength & $\mathbf{R}^{\mathbf{2}}$ Value \\
\hline & $\sigma$ & $M$ & $\sigma_{0}$ & $\mathrm{R}^{\mathbf{2}}$ \\
\hline & $(\mathrm{MPa})$ & (Dimensionless) & $(\mathrm{MPa})$ & $($ Dimensionless $)$ \\
\hline $9-043$ & 200.8 & 5 & 218.1 & 0.98 \\
\hline $9-047$ & 225.9 & 7 & 241.3 & 0.97 \\
\hline $9-063$ & 177.3 & 4 & 194.2 & 0.96 \\
\hline $9-080$ & 206.8 & 5 & 224.8 & 0.83 \\
\hline $9-081$ & 189.4 & 4 & 209.5 & 0.92 \\
\hline $9-083$ & 237.1 & 7 & 252.1 & 0.97 \\
\hline $9-140$ & 240.3 & 7 & 256.1 & 0.96 \\
\hline $9-152$ & 169.7 & 2 & 202.7 & 0.87 \\
\hline $9-188$ & 270.7 & 8 & 286.9 & 0.98 \\
\hline $9-193$ & 237.6 & 8 & 252.2 & 0.91 \\
\hline $9-203$ & 226.8 & 5 & 247.9 & 0.94 \\
\hline $9-221$ & 243.9 & 10 & 255.9 & 0.91 \\
\hline $9-224^{*}$ & 301.0 & 9 & 317.5 & 0.95 \\
\hline
\end{tabular}


Table A7. Summary of Weibull Data for the 14 Pipe Samples Collected Under The Stratified Sampling Programme for High Burst Rate DMA, Zero Burst Rate Streets.

\begin{tabular}{|c|c|c|c|c|}
\hline TWA Sample Ref. & Average Strength & Weibull Modulus & Char. Strength & $\mathbf{R}^{\mathbf{2}}$ Value \\
\hline & $\sigma$ & $m$ & $\sigma_{0}$ & $\mathrm{R}^{\mathbf{2}}$ \\
\hline & $(\mathrm{MPa})$ & $($ Dimensionless $)$ & $(\mathrm{MPa})$ & $($ Dimensionless $)$ \\
\hline $9-044$ & 130.5 & 1 & 152.6 & 0.89 \\
\hline $9-046$ & 247.6 & 12 & 258.1 & 0.96 \\
\hline $9-109$ & 240.3 & 4 & 265.3 & 0.92 \\
\hline $9-115^{*}$ & 201.8 & 5 & 218.9 & 0.95 \\
\hline $9-116$ & 146.1 & 3 & 166.4 & 0.92 \\
\hline $9-121$ & 182.4 & 3 & 207.6 & 0.91 \\
\hline $9-122$ & 234.3 & 5 & 256.1 & 0.95 \\
\hline $9-155$ & 176.1 & 3 & 197.4 & 0.95 \\
\hline $9-169$ & 222.4 & 3 & 249.6 & 0.89 \\
\hline $9-170$ & 277.6 & 7 & 296.9 & 0.95 \\
\hline $9-178$ & 234.6 & 9 & 247.1 & 0.91 \\
\hline $9-184^{*}$ & 556.8 & 14 & 576.6 & 0.97 \\
\hline $9-229$ & 327.5 & 19 & 336.5 & 0.77 \\
\hline $9-249$ & 271.9 & 7 & 290.3 & 0.99 \\
\hline
\end{tabular}

